Article

\title{
Exploratory Analysis of Lenvatinib Therapy in Patients with Unresectable Hepatocellular Carcinoma Who Have Failed Prior PD-1/PD-L1 Checkpoint Blockade
}

\author{
Tomoko Aoki 1,+(D), Masatoshi Kudo ${ }^{1, *,+} \mathbb{D}$, Kazuomi Ueshima ${ }^{1}$, Masahiro Morita ${ }^{1}$, \\ Hirokazu Chishina ${ }^{1}$, Masahiro Takita ${ }^{1}$, Satoru Hagiwara ${ }^{1}$, Hiroshi Ida $^{1}$, Yasunori Minami ${ }^{1}$ (D), \\ Masakatsu Tsurusaki ${ }^{2}$ (D) and Naoshi Nishida ${ }^{1}$ (D) \\ 1 Department of Gastroenterology and Hepatology, Kindai University Faculty of Medicine, \\ 377-2 Ohno-higashi, Osaka-Sayama, Osaka 589-8511, Japan; t.aoki1918@gmail.com (T.A.); \\ kaz-ues@med.kindai.ac.jp (K.U.); s0750081@yahoo.co.jp (M.M.); chishina@med.kindai.ac.jp (H.C.); \\ masahirot2797@yahoo.co.jp (M.T.); hagi-318@hotmail.co.jp (S.H.); hidakuhp@gmail.com (H.I.); \\ minkun@med.kindai.ac.jp (Y.M.); naoshi@med.kindai.ac.jp (N.N.) \\ 2 Department of Radiology, Kindai University Faculty of Medicine, 377-2 Ohno-higashi, \\ Osaka-Sayama, Osaka 589-8511, Japan; mtsuru@dk2.so-net.ne.jp \\ * Correspondence: m-kudo@med.kindai.ac.jp; Tel.: +81-72-366-0221 (ext. 3149) \\ + These authors contributed equally to this manuscript.
}

Received: 15 September 2020; Accepted: 8 October 2020; Published: 20 October 2020

check for updates

Simple Summary: Programmed cell death protein 1 (PD-1)/PD-ligand 1 (PD-L1) blockade is becoming a novel therapeutic option for a hepatocellular carcinoma. In this work, we evaluated efficacy and safety of lenvatinib following failure of PD-1/PD-L1 blockade. The median progression-free survival was 10 months (95\% confidence interval (CI): 8.3-11.8) and the median overall survival was 15.8 months (95\% CI: 8.5-23.2) since lenvatinib therapy initiation. The objective response rate was $55.6 \%$, and the disease control rate was $86.1 \%$. All of efficacy outcomes were better than those by lenvatinib treatment alone as the 1st line treatment therapy. No particular safety concerns were observed. It was speculated that lenvatinib right after failure of PD-1/PD-L1 blockade provided synergistic effect since anti-PD-1 antibodies can remain binding to CD8+T cells for more than several months. Lenvatinib demonstrated considerably high antitumor activity and good survival benefit with acceptable toxicity in patients with unresectable HCC when administered right after failure of PD-1/PD-L1 blockade.

\begin{abstract}
Although programmed cell death protein 1 (PD-1)/PD-ligand 1 (PD-L1) blockade is effective in a subset of patients with hepatocellular carcinoma (HCC), its therapeutic response is still unsatisfactory. Alternatively, the potential impact of the lenvatinib in patients who showed tumor progression on PD-1/PD-L1 blockade is unknown. In this work, we evaluated the safety and efficacy of lenvatinib administration after PD-1/PD-L1 checkpoint blockade. The outcome and safety of lenvatinib administered after PD-1/PD-L1 blockade failure was analyzed retrospectively in 36 patients. Tumor growth was assessed every $4-8$ weeks using modified Response Evaluation Criteria in Solid Tumors. The mean relative dose intensity of lenvatinib was $87.6 \%$ and $77.8 \%$ in patients receiving a starting dose of 8 (interquartile range (IQR), 77.5-100.0) $\mathrm{mg}$ and 12 (IQR, 64.4-100.0) $\mathrm{mg}$, respectively. Since lenvatinib therapy initiation, the median progression-free survival was 10 months (95\% confidence interval (CI): 8.3-11.8) and the median overall survival was 15.8 months (95\% CI: 8.5-23.2). The objective response rate was $55.6 \%$, and the disease control rate was $86.1 \%$. No particular safety concerns were observed. Lenvatinib demonstrated considerable antitumor effects with acceptable safety in patients with progressive and unresectable HCC when administered right after PD-1/PD-L1 blockade failure.
\end{abstract}


Keywords: hepatocellular carcinoma; lenvatinib; PD-1/PD-L1 blockade; molecular targeted agents; vascular endothelial growth factor

\section{Introduction}

Hepatocellular carcinoma (HCC) is the most common primary malignancy of the liver and one of the major causes of cancer-related death worldwide [1-5]. Unfortunately, owing to the late occurrence of symptoms, HCC progression is often diagnosed at an advanced state, which prevents the application of locoregional therapies. Even when patients diagnosed with early or intermediate tumor stages are treated, prognosis is poor because HCC has a high recurrence rate. Considering the high number of HCC cases with progressive disease (PD), the development of an effective systemic therapy has been an urgent need over the past decade for the management of unresectable tumors.

Recently, targeting unique signaling pathways critical in tumor growth has emerged as a promising strategy for HCC treatment. In this context, several molecular targeted agents (MTAs), including sorafenib [6,7], lenvatinib [8], regorafenib [9], ramucirumab [10], and cabozantinib [11], have been used in clinical settings [12,13]. Notably, lenvatinib is a multikinase inhibitor that targets vascular endothelial growth factor (VEGF) receptors 1-3, platelet-derived growth factor (PDGF) receptor alpha, fibroblast growth factor (FGF) receptors 1-4, rearranged during transfection (RET), and KIT, which draws a strong anti-tumor response in HCC cases [14-17].

Alternatively, immunotherapy is becoming a novel therapeutic option for a variety of cancers [18-20]. Antibodies against programmed cell death protein 1 (PD-1) have shown prolonged antitumor responses in patients with advanced HCC [21,22]. For this reason, the Food and Drug Administration ( $w w w . f d a . g o v)$ approved the anti-PD-1 antibodies nivolumab [23] and pembrolizumab [24] as second-line therapies following sorafenib treatment. However, phase III clinical trials failed to show the survival benefits of these agents for the treatment of unresectable HCC in both first- and second-line settings $[25,26]$. On the other hands, in patients with unresectable HCC, atezolizumab combined with bevacizumab resulted in better overall and progression-free survival outcomes than sorafenib [27], so combination therapy of anti-VEGF/MTA and anti-PD-1/PD-L1 blockade might be new first-line treatment option for patients with unresectable HCC instead of sorafenib or lenvatinib.

Although a precise mechanism underlying the refractoriness to immune checkpoint inhibitors (ICIs) in patients with HCC remains unexplored, hormones, such as vascular endothelial growth factor (VEGF), could mediate such refractoriness. VEGF secreted by HCC cells could result in ICI resistance by inducing $\mathrm{CD}^{+} \mathrm{T}$ cell exhaustion and an immunosuppressive cellular phenotype in the tumor microenvironment (TME) [28,29].It has also been reported that compared with HCC without activating mutations the Wnt/ $\beta$-catenin pathway, HCC with such mutations can induce an alteration in immune cell recruitment and result in poorer prognosis following ICI treatment. This suggests a role of the Wnt/ $\beta$-catenin pathway in the establishment of "immune cold" phenotype in HCC [30].

In contrast, considering the potential anti-VEGF activity of MTAs, these agents could alter the immunological microenvironment and reverse the refractoriness to ICIs. In addition, few MTAs exert their antitumor effect through a VEGF-independent mechanism by inhibiting the signaling pathways involved in tumor growth. In this scenario, the combination of ICIs and MTAs could be an attractive option for the treatment of advanced HCC cases and even in those refractory to ICI monotherapies [31]. Indeed, the efficacy of atezolizumab and bevacizumab [27] or pembrolizumab and lenvatinib [32] combination has been confirmed, although no real-world data are available for such combinations for HCC treatment. In contrast, as antibodies are considerably stable in the living body [33], it is reasonable to expect that sequential therapy with MTAs following anti-PD-1 antibody will exert a synergic effect similar to their combinatorial therapy. Among the MTAs available for HCC, lenvatinib showed the 
most potent antitumor activity [34], and it would be intriguing to evaluate its efficacy in HCC refractory to ICI monotherapy.

Thus, we present here the first real-world data analysis of lenvatinib efficacy and safety following the clinical trial of PD-1/PD-L1 blockade in patients with HCC unresponsive to ICIs. Although this study is conducted as a sequential therapy not a combination, anti-PD-1 antibody is known to bind on $\mathrm{CD}^{+} \mathrm{T}$ cells for more than 20 months, where sustained blockade to PD-1 can be expected [32]. In addition, inhibitory activity of lenvatinib against the FGF signaling pathway of HCC is prominent, considering the low Median Inhibition Concentration $\left(\mathrm{IC}_{50}\right)$ value for fibroblast growth factor receptor 4 (FGFR4) [35]. From this point of view, lenvatinib could be effective for HCC with high FGFR4 expression that prone to carry Wnt/ $\beta$-Catenin mutation [36]. Collectively, it is conceivable that ICI-lenvatinib sequential therapy would be also effective, similar with their combination, which is the clinical question to be clarified in this exploratory analysis.

\section{Results}

\subsection{Patient Characteristics}

A total of 170 patients received lenvatinib at our institution. Between March 2016 and September 2019, 36 of these patients who received lenvatinib as a next-line treatment immediately after anti-PD-1/PD-L1 therapy failure were enrolled in the present analysis. Of these 36 patients, 14 received a systemic therapy before ICI therapy. Patient characteristics have been summarized in Table 1. The median age of patients was 70 (range, 60.8-76.8) years, and most patients were male (31.86\%), with a male to female ratio of $6: 1$. In this cohort, $55.6 \%(20 / 36)$ of the patients were negative for hepatitis B surface antigen (HBsAg) and hepatitis C antibody (HCVAb), 27.8\% (10/36) were positive $\mathrm{HCVAb}$, and 16.7\% (6/36) were positive for HBsAg. All patients had Child-Pugh grade A liver cirrhosis. The tumors were large and/or multi-nodular in all patients, where $25 \%$ of the patients (9/36) were Barcelona Clinic Liver Cancer (BCLC)-stage B [37] and 75\% (27/36) of the patients were BCLC-stage C. Vascular invasion and extrahepatic spread were observed in $13.9 \%$ and $72.2 \%$ patients, respectively. The median $\alpha$-fetoprotein (AFP) level was 76.5 (interquartile range (IQR), 5.25-11,543.5) $\mathrm{ng} / \mathrm{mL}$, with $44.4 \%$ of patients having an AFP higher than $400 \mathrm{ng} / \mathrm{mL}$. The median des- $\gamma$-carboxy prothrombin (DCP) level was 1303.5 (IQR, 43.25-7394.3) milli arbitrary units per milliliter (mAU/mL), with $63.9 \%$ of the patients having a DCP higher than $100 \mathrm{mAU} / \mathrm{mL}$. The duration of prior PD-1/PD-L1 checkpoint blockade ranged from 1.7 to 8.8 (median, 3.7) months.

Table 1. Baseline characteristics of patients.

\begin{tabular}{cc}
\hline Characteristics & Overall $(\boldsymbol{n}=\mathbf{3 6})$ \\
\hline Age, median & $70(60.75-76.75)$ \\
Gender, male/female & $31 / 5$ \\
Etiology: HCV/HBV/NBNC & $10 / 6 / 20$ \\
Albumin, median (g/dL) & $3.800(3.625-4.100)$ \\
Total bilirubin, median $(\mathrm{mg} / \mathrm{dL})$ & $0.600(0.500-0.975)$ \\
ALBI score, median & $-2.590(-2.827--2.267)$ \\
FIB4 index, median & $2.778(1.644-5.191)$ \\
BCLC stage, B/C & $9 / 27$ \\
Vascular invasion, with/without & $5 / 31$ \\
Extrahepatic spread, with/without & $26 / 10$ \\
Baseline AFP, median $(\mathrm{ng} / \mathrm{mL})$ & $76.5(5.25-11543.5)$ \\
DCP, median $(\mathrm{mAU} / \mathrm{mL})$ & $1303.5(43.25-7394.3)$ \\
\hline
\end{tabular}

$\mathrm{HCV}$, hepatitis C virus; HBV, hepatitis B virus; NBNC, HBV and HCV negative; BCLC, Barcelona Clinic Liver Cancer; ALBI grade, albumin-bilirubin grade; FIB4, The fibrosis-4 (FIB-4) index was calculated as age (year) $\times$ AST $(\mathrm{IU} / \mathrm{L}) /($ platelet count $(109 / \mathrm{L}) \times \sqrt{ }$ ALT $(\mathrm{IU} / \mathrm{L}))$ [38]; AST, aspartate aminotransferase; ALT, alanine aminotransferase; AFP, $\alpha$-fetoprotein concentration; DCP, Des- $\gamma$-carboxy prothrombin. We express the inspection value in Median (interquartile range). 


\subsection{Administration of Lenvatinib and Outcomes}

At the end of the study period (29 February 2020), the median follow-up duration was 5.5 (range, 1.1-20) months. During the observation period, 11 patients died because of HCC progression. The mean duration between ICI therapy termination and lenvatinib treatment initiation was 3.65 (IQR, 0.50-4.46) months, with a median treatment period of 4.6 (IQR, 2.2-10.0) months. The mean relative dose intensity for lenvatinib with starting doses of $8 \mathrm{mg}$ and $12 \mathrm{mg}$ was $87.6 \%$ (IQR, 77.5-100.0\%) and $77.8 \%$ (IQR, 64.4-100.0\%), respectively. The median time for lenvatinib first dose reduction was 6.3 (IQR, 2.4-26.0) weeks. Treatment was discontinued in 15 patients owing to disease progression $(n=10,27.8 \%)$ or treatment-related adverse events (AEs) $(n=5,13.9 \%)$. Fourteen ( $39 \%)$ of the 36 patients with a response are under the ongoing responses.

\subsection{Antitumor Response and Patient Survival After Lenvatinib Administration Following Immune Checkpoint Inhibitor Therapy}

The median progression-free survival (PFS) was 10.0 months (95\% confidence interval (CI): 8.3-11.8, Figure 1), and the median overall survival (OS) calculated since the initiation of lenvatinib therapy was 15.8 months (95\% CI: 8.5-23.2, Figure 2). The median OS since ICI therapy initiation was 29.8 months (95\% CI: 25.3-34.4). The objective response rate (ORR) and disease control rate (DCR) in patients who underwent radiological evaluation were $55.6 \%$ and $86.1 \%$, respectively, as assessed using the modified Response Evaluation Criteria in Solid Tumors (mRECIST) [39]. Complete response (CR), partial response (PR), stable disease (SD), and PD were noted in 2.8\% ( $n=1), 52.8 \%(n=19), 30.6 \%(n=11)$, and $11.1 \%(n=4)$ patients, respectively. The ORR and DCR, evaluated using RECISTv1.1, were $22.2 \%$ $(n=8)$ and $94.4 \%(n=34)$, respectively, whereas CR, PR, SD, PD were $0.0 \%(n=0), 22.2 \%(n=8)$, $72.2 \%(n=26)$, and $0.0 \%$, respectively.

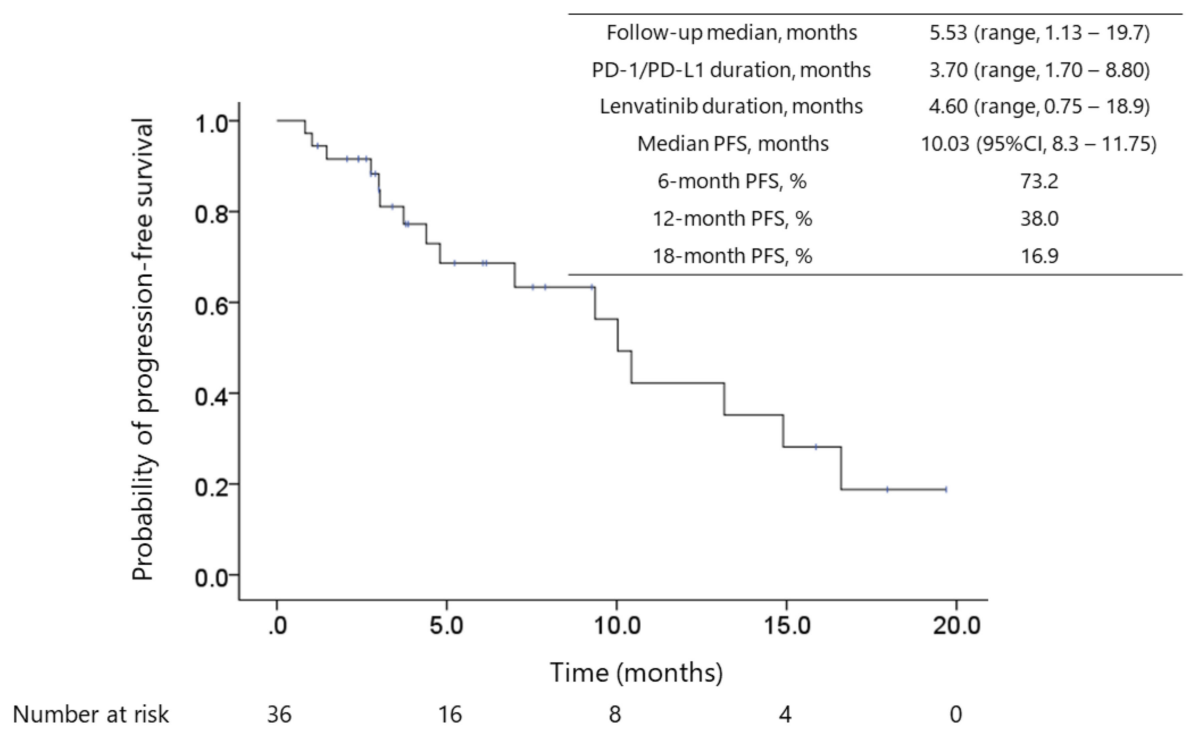

Figure 1. Kaplan-Meier survival curve of progression-free survival (PFS). The median duration of prior PD-1/PD-L1 checkpoint blockade treatment, between ICI termination and lenvatinib treatment initiation, and lenvatinib treatment was 3.7 (IQR, 1.7-8.8) months, 0.95 (IQR, 0.55-4.08) months, and 4.6 (IQR, 2.2-10) months, respectively. Fourteen (39\%) patients continued the treatment with complete or partial response status. The median progression-free survival (PFS) was 10 months (95\%CI: 8.3-11.8) for 36 patients. Of these, the median PFS was not reached for 22 patients who were administered lenvatinib as the 2nd-line systemic therapy, and the median PFS was 10.4 months (95\% CI: 9.08-11.8) for 14 patients who received prior systemic therapy before ICI treatment. 


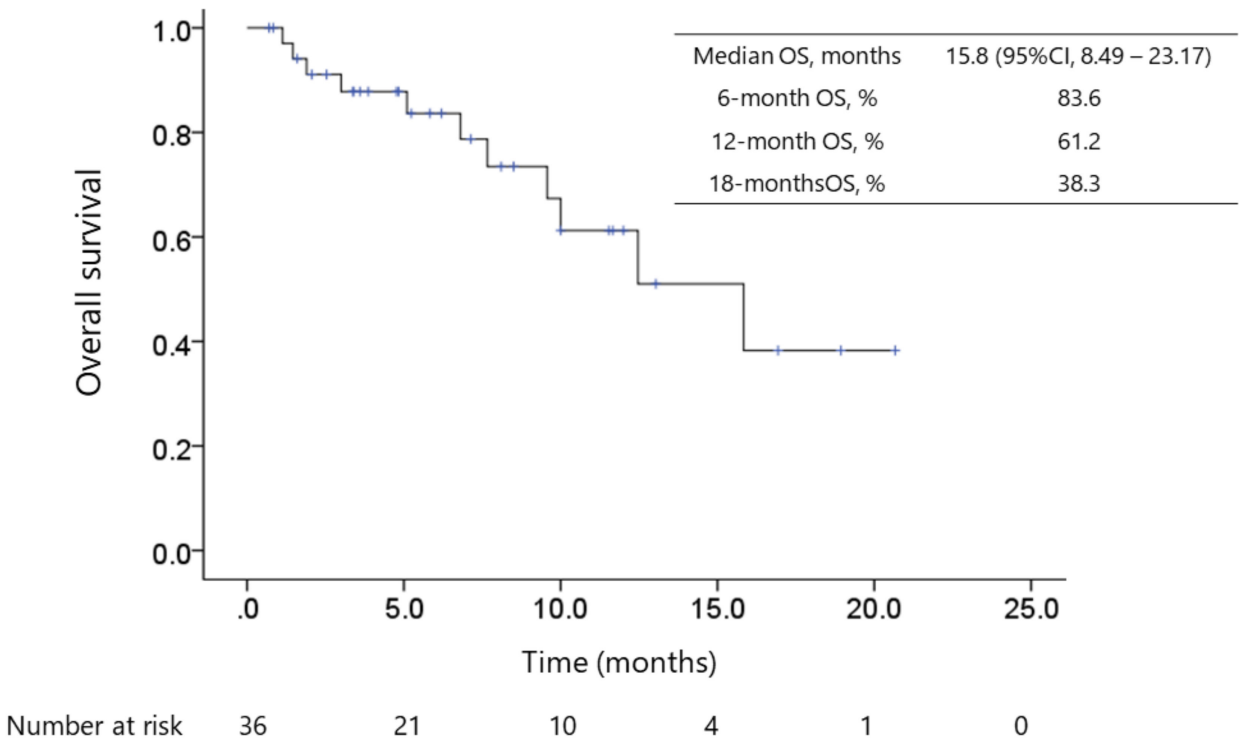

Figure 2. Kaplan-Meier survival curve of overall survival (OS). The median follow-up duration was 5.53 (IQR 2.88-10.38) months and 11 patients died of HCC progression. The median periods of OS since the initiation of lenvatinib therapy and since ICI therapy initiation were 15.8 months $(95 \% \mathrm{CI}$ : 8.5-23.2) and 29.8 months (95\% CI: 25.3-34.4), respectively, for 36 patients.

Early responses of lenvatinib were observed during the first 4 weeks in 30 out of 36 patients $(83.3 \%)$. Of these, only 4 patients showed tumor regrowth at the 8 th week, compared with the tumor size at the baseline. This suggested a persistent tumor response to this treatment (Figure 3). The median maximum tumor response, i.e., depth of response, was $-41.2 \%(\mathrm{IQR},-23.8--61.8)$ in 15 patients $(75 \%)$ out of 20 responsive patients (Figure 4).

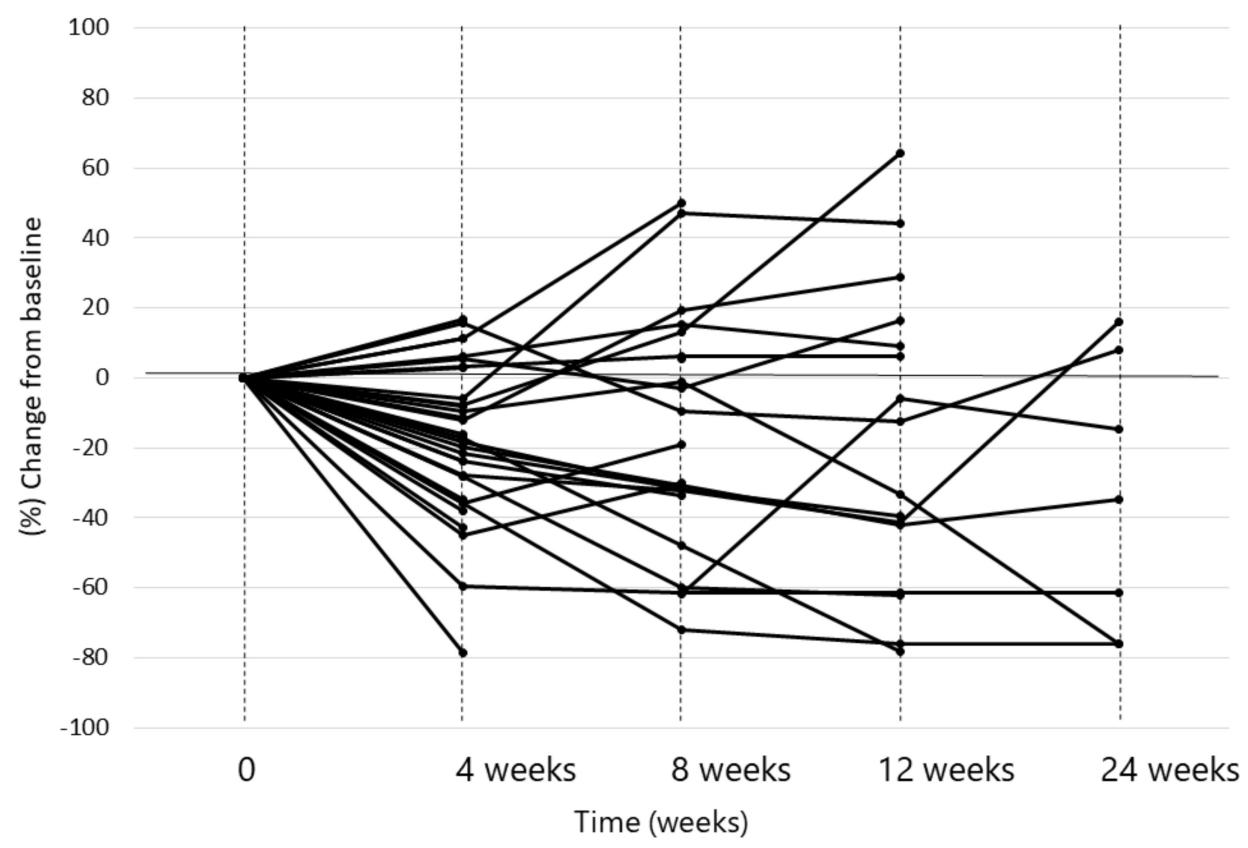

Figure 3. Spider-plot of the HCC cases who were treated with lenvatinib after the failure of immune checkpoint blockade. Decreases of tumor size were observed at the 4 th week of the treatment in 30 out of 36 patients $(83.3 \%)$. Of these, only 4 patients showed increase of tumor size at the 8 th week, compared with that at the baseline. 


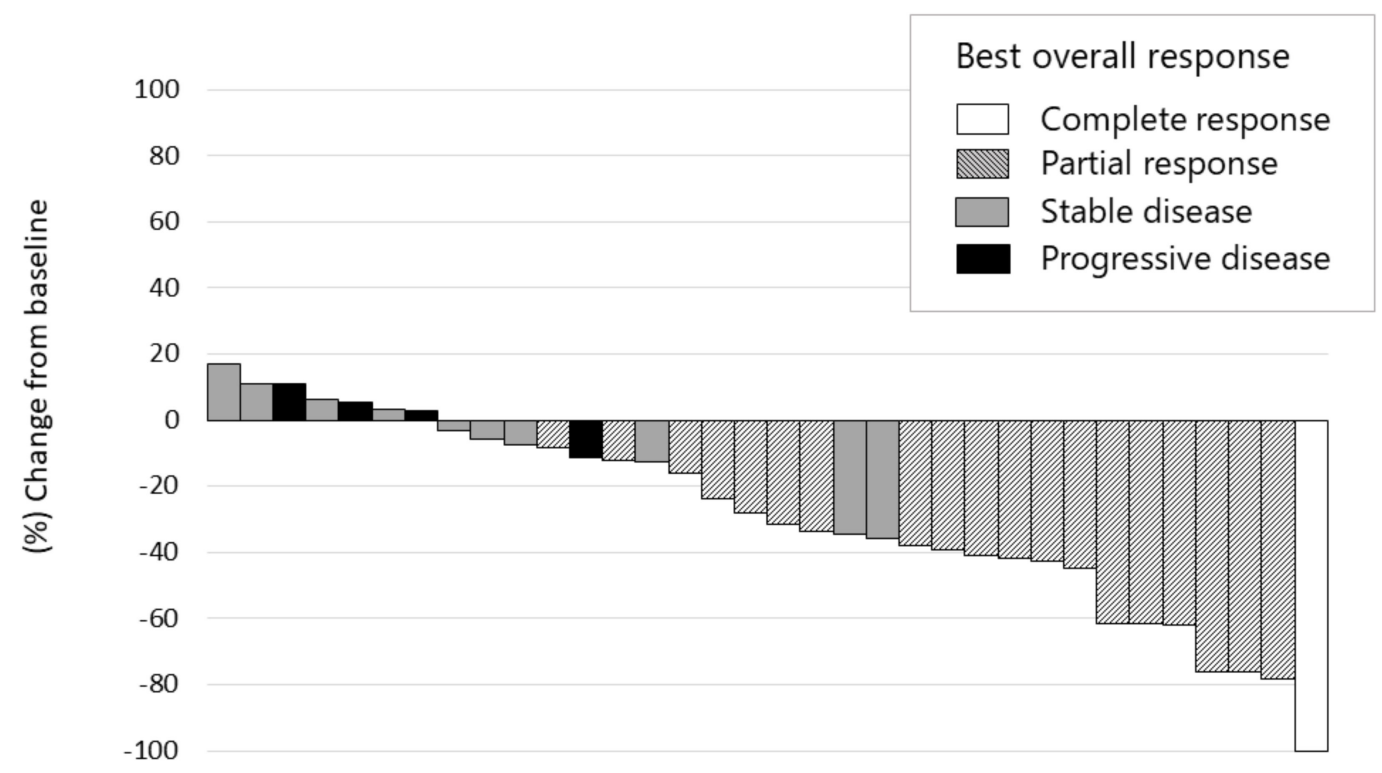

Figure 4. Waterfall plot of maximum tumor response to lenvatinib therapy using mRECIST. Complete response, partial response, stable disease, and progressive disease were noted in $2.8 \%(n=1)$, $52.8 \%(n=19), 30.6 \%(n=11)$, and $11.1 \%(n=4)$, respectively. The objective response rate and disease control rate in patients who underwent radiological evaluation were $55.6 \%$ and $86.1 \%$, respectively, as assessed using the mRECIST. The depth of response was $-25.9 \%$ (IQR $-43.0--5.2)$ in all patients and $-41.2 \%$ (IQR -61.8--23.8) in responders.

\subsection{Safety Outcomes}

The adverse events (AEs) of any grade observed are shown in Table 2. AEs were observed in all patients, with grade 3 or 4 AEs occurring in $20(55.6 \%)$ patients. The most common AE was liver dysfunction (21 patients, 58.3\%) and was mostly grade 2 or lower. Hypertension was the second most common AE (16 patients, 44.4\%) and was easily treated with an antihypertensive therapy. Diarrhea, anorexia, and other gastrointestinal symptoms occurred in $15(41.7 \%)$ patients, and hand-foot syndrome was noted in $9(25.0 \%)$ patients. Anorexia and/or malaise was the most common reason for treatment discontinuation and occurred in $13.9 \%$ of patients.

Table 2. Adverse events observed in this study.

\begin{tabular}{ccc}
\hline Adverse Events & Any Grade & Grade 3/4 \\
\hline Liver dysfunction & $21(58.3 \%)$ & $3(8.3 \%)$ \\
Hypertension & $16(44.4 \%)$ & $4(11.1 \%)$ \\
Decreased appetite & $15(41.7 \%)$ & $1(2.8 \%)$ \\
Diarrhea & $15(41.7 \%)$ & $3(8.3 \%)$ \\
Fatigue & $13(36.1 \%)$ & $1(2.8 \%)$ \\
Hypothyroidism & $12(33.3 \%)$ & $0(0 \%)$ \\
Hands foots skin reaction & $9(25 \%)$ & $1(2.8 \%)$ \\
Hypoalbuminaemia & $9(25 \%)$ & $1(2.8 \%)$ \\
Jaundice & $8(22.2 \%)$ & $2(5.6 \%)$ \\
Hoarseness & $7(19.4 \%)$ & $0(0 \%)$ \\
Thronbocytopenia & $6(16.7 \%)$ & $3(8.3 \%)$ \\
Infection & $5(13.9 \%)$ & $2(5.6 \%)$ \\
Encephalopathy & $3(8.3 \%)$ & $1(2.8 \%)$ \\
Peripheral edema & $3(8.3 \%)$ & $0(0 \%)$ \\
Bleeding or haemorrhage & $2(5.6 \%)$ & $1(2.8 \%)$ \\
Proteinuria & $2(5.6 \%)$ & $0(0 \%)$ \\
Ascites & $1(2.8 \%)$ & $0(0 \%)$ \\
\hline
\end{tabular}




\section{Discussion}

A combination of MTAs and ICIs for treating unresectable HCC could represent a promising strategy as these agents act in a complementary manner to induce a tumor response through different antitumor mechanisms. In particular, anti-VEGF agents are ideal for use in combination with ICI treatments as VEGF plays a potential role in establishing an immunosuppressive TME [31,40]. Indeed, the combination of bevacizumab with anti-PD-L1 antibody or lenvatinib with anti-PD-1 antibody has shown considerable antitumor response [32]. However, these combinations have not been used in clinical settings yet. Considering the prolonged activity of these antibodies [33], we can expect similar therapeutic efficacy with lenvatinib and ICI combination therapy when lenvatinib is administered after ICI treatment. This is because anti-PD-1/PD-L1 antibodies administrated prior to lenvatinib might be active even after the initiation of lenvatinib administration.

In this work, we confirmed the efficacy and safety of lenvatinib in patients with HCC who were unresponsive to PD-1/PD-L1 blockade. Approximately $70-80 \%$ of patients with $\mathrm{HCC}$ are refractory to ICI monotherapy, suggesting the presence of potential resistance mechanisms against these agents during tumor progression $[23,24,27]$. This justifies an urgent need to establish an effective regimen targeting the antitumor immunity in patients with HCC refractory to ICIs. Our results showed high tumor response rates (ORR: 55.6\%; DCR: $86.1 \%$ ) and a relatively high proportion of patients who sustained a tumor response. Moreover, no rapid, aggressive HCC progression was observed in this study.

The median PFS of 10 months observed in our study was longer than that observed in the lenvatinib cohort of REFLECT trial (7.4 months) [8]. However, it should be noted that the REFLECT trial was a phase III study comparing lenvatinib with sorafenib as a first-line treatment for patients with unresectable HCC (Table 3). However, in our cohort, lenvatinib was used after ICI therapy as a second-, third-, and fourth-line systemic treatment in 22 (61.1\%), $9(25.0 \%)$, and 5 (13.9\%) patients, respectively. For this reason, the presented data are quite interesting, and they indicate a potential advantage of administering lenvatinib to patients with HCC who have been previously treated with ICIs. In line with this evidence, a recent phase III clinical trial reported a median PFS of 6.8 months (95\% CI: 5.7-8.3) with an ORR of 33.2\% (95\% CI: 28.1-38.6) in patients treated with a combination of atezolizumab and bevacizumab immunotherapy using mRECIST [27]. In addition, a phase I b study that assessed lenvatinib plus pembrolizumab therapy in patients with HCC showed a median PFS of 9.3 months (95\% CI: 5.6-9.7) with an ORR of 46.0\% (95\% CI: 36.0-56.3) using mRECIST [32]. Therefore, lenvatinib administration after ICI therapy showed comparable antitumor response and survival with anti-VEGF agent plus ICI combination therapy (Table 3). It has been reported that anti-PD-1 antibodies can remain bound to $\mathrm{CD} 8^{+} \mathrm{T}$ cells for more than 20 weeks [33]. Therefore, it is quite reasonable to speculate that during lenvatinib treatment, previously administered anti-PD-1/PD-L1 antibody treatment exerts an effect on immune cells as well as tumor cells and induces a synergistic effect with lenvatinib, similar to that observed in the combination therapy of MTAs with ICIs. Further analysis should be conducted in this regard by analyzing the effect of anti-PD-1/PD-L1 antibody on CD8 ${ }^{+} \mathrm{T}$ cells in patients with HCC.

Overcoming the refractoriness to ICI represents a major challenge to improve the prognosis of patients with cancer including HCC. There are several factors associated with ICI resistance: loss of expression of PD-1/PD-L1 in tumor and antigen-presenting cells [23,41]; low mutation burden limiting tumor associated antigen expression; canonical Wnt $/ \beta$-catenin pathway activation affecting immune-mediated cell infiltration [42-44]; $\beta-2$ microglobulin mutations impairing antigen presentation $[35,45,46]$; and alterations in the janus kinase-signal transducers and activators of transcription pathway affecting interferon- $\gamma$ signaling [36]. Harding et al. [42] have reported that the activating alterations of Wnt $/ \beta$-catenin signaling are associated with lower DCR, shorter median PFS, and shorter median OS in patients with HCC treated with ICIs. Furthermore, the expression of multiple repressive immune checkpoint receptors, such as $\mathrm{T}$ lymphocyte, including cytotoxic $\mathrm{T}$ 
lymphocyte-associated antigen 4, lymphocyte-activation gene 3, or T-cell immunoglobulin mucin-3, could also be associated with impaired responses to PD-1/PD-L1 blockade.

Table 3. Comparison of anti-tumor response of lenvatinib after PD-1/PD-L1 with that of lenvatinib monotherapy, and anti-VEGF and anti-PD-1 blockade combinations.

\begin{tabular}{|c|c|c|c|c|}
\hline $\begin{array}{c}\text { Study } \\
\text { Name/ID }\end{array}$ & REFLECT [8] & Presented Study & NCT03006926 & NCT03434379 \\
\hline Treatment & Monotherapy & Sequential & \multicolumn{2}{|c|}{ Combination } \\
\hline Agents & Lenvatinib & $\begin{array}{l}\text { Lenvatinib after } \\
\text { PD-1/PD-L1 }\end{array}$ & Lenvatinib + Pembrolizumab & Atezolizumab + Bevacizumab \\
\hline \multicolumn{5}{|l|}{ Condition } \\
\hline Design & Phase III & Retrospective & Phase I b & Phase III \\
\hline $\begin{array}{l}\text { Number of } \\
\text { patents }\end{array}$ & 478 & 36 & 100 & 336 \\
\hline Setting & 1st line & 2-4th line & 1st line & 1st line \\
\hline \multicolumn{5}{|l|}{ Outcome } \\
\hline $\begin{array}{c}\text { ORR } \\
\text { (mRECIST) }\end{array}$ & $24.1 \%$ & $55.6 \%$ & $46.0 \%$ & $33.2 \%$ \\
\hline $\begin{array}{c}\text { DCR } \\
(\mathrm{mRECIST})\end{array}$ & $73.8 \%$ & $86.1 \%$ & $86.0 \%$ & $72.3 \%$ \\
\hline median PFS & 7.4 months & 10 months & 9.3 months & 6.8 months \\
\hline \multirow[t]{2}{*}{ median OS } & 13.6 months & 15.8 months & 22 months & (12 months, OS $67.2 \%$ ) \\
\hline & & $\begin{array}{l}\text { (29.8 months since } \\
\text { ICIs started) }\end{array}$ & & \\
\hline \multirow{8}{*}{ Adverse events } & HT $42 \%$ & HT $44 \%$ & HT $36 \%$ & HT $30 \%$ \\
\hline & diarrhea 39\% & diarrhea $42 \%$ & diarrhea 35\% & diarrhea 19\% \\
\hline & $\begin{array}{c}\text { appetite loss } \\
34 \%\end{array}$ & appetite loss $42 \%$ & fatigue $30 \%$ & fatigue $20 \%$ \\
\hline & weight loss $31 \%$ & fatigue $36 \%$ & AST increase $20 \%$ & AST increase $20 \%$ \\
\hline & $\begin{array}{c}\text { AST increase } \\
14 \%\end{array}$ & AST increase $58 \%$ & & \\
\hline & \multicolumn{4}{|c|}{ any grade $99 \%$} \\
\hline & any grade $99 \%$ & any grade $100 \%$ & grade $3,85 \%$ & Any grade $98 \%$ \\
\hline & grade 3,$4 ; 75 \%$ & grade 3,$4 ; 56 \%$ & grade $4,23 \%$ & Grade 3,$4 ; 57 \%$ \\
\hline
\end{tabular}

ORR, objective response rate; DCR, disease control rate; PFS, progression-free survival; OS, overall survival; mRECIST, modified Response Evaluation Criteria in Solid Tumors; ICI, immune checkpoint inhibitor; PD-1, programmed cell death protein 1; PD-L1, PD-ligand 1; HT, hypertension; AST, aspartate aminotransferase. NCT03006926 [32] and NCT03434379 [27].

Another interesting report showed that $\beta$-catenin mutation correlates very well with increased expression of FGFR4 [36]. As lenvatinib has a strong inhibitory effect of FGFR4 signaling pathway with $\mathrm{IC}_{50}$ to FGFR4 of $43 \mathrm{nM} / \mathrm{L}$, [47] higher response rate $(81 \%$ vs. $31 \%)$ and better PFS (5.5 M vs. $\left.2.5 \mathrm{M}\right)$ were observed in HCC patients with high FGFR4 expression (positive $\beta$-catenin activation) than those with low FGFR4 expression [48]. This might be another explanation of better efficacy of lenvatinib after failure of PD-1/PD-L1 antibody therapy.

Reportedly, VEGF is known as an immunosuppressive growth factor, and its increased level induces the recruitment and activation of immunosuppressive cells expressing VEGF receptors [49]. As VEGF is secreted by HCC cells, it could play a critical role in determining the insufficient immune response noted in patients with HCC. Considering the mutational profile and pathophysiology of HCC, VEGF-associated immunosuppressive TME could be a possible cause of ICI refractoriness in patients with HCC. Indeed, recent data from a phase III clinical trial revealed a higher response rate with anti-VEGF-A and anti-PD-L1 combinatorial therapy than with PD-1/PD-L1 blockade monotherapy. In line with these data, Shigeta et al. reported the occurrence of synergistic antitumor effects of the combination of anti-PD-1 and anti-VEGFR-2 antibodies using murine models of HCC [50]. Combination 
therapy reprogrammed the TME by increasing $\mathrm{CD} 8^{+}$cytotoxic $\mathrm{T}$ cell infiltration and activation, shifting the M1/M2 ratio of tumor-associated macrophages with reduction of T regulatory cell and infiltration of C-C chemokine receptor 2-positive monocyte in HCC tissue [50]. From this standpoint, the antitumor mechanism of lenvatinib administered after ICI therapy could be, at least partially, attributed to the targeting of the immunosuppressive cells expressing VEGF receptors and the restoring of the residual anti-PD-1/PD-L1 antibody function of antitumor $\mathrm{CD} 8^{+}$cells, in addition to the targeting of tumor cells [51].

There are several anti-VEGF/MTA agents already available for use in clinical settings and for easy use in ICI-based therapy. Lenvatinib administration following anti-PD-1/PD-L1 blockade failure showed stronger antitumor response than that shown by a previously reported ICI monotherapy or lenvatinib monotherapy. Moreover, it showed clinical efficacy similar to that shown by combination therapies. Besides, the safety profile of lenvatinib was generally consistent with that reported in REFLECT trial. Most lenvatinib-related AEs were generally mild and tended to resolve on treatment continuation, without any serious adverse events. This also contributed to the long-term administration and considerable efficacy of lenvatinib.

Despite the promising results, our study has several limitations. First, this was the preliminary retrospective study in nature with no supporting data from comparative studies. Although the number of patients is small, we were able to obtain results that would be a proof of concept, i.e., sequential therapy of ICI-lenvatinib is more effective than lenvatinib alone as an exploratory analysis, so it is warranted to confirm whether our result is correct by conducting prospective randomized study in the future. Second, a selection bias could exist because of the clinical observational nature of the study. Nevertheless, this real-world data analysis indicates high efficacy of lenvatinib therapy following ICI treatment failure, which indicates that lenvatinib can be strongly recommended in cases refractory to ICI monotherapy.

\section{Materials and Methods}

\subsection{Patients}

This single-institute, retrospective study enrolled patients with unresectable advanced HCC who received PD-1/PD-L1 checkpoint blockade followed by lenvatinib from 1 March 2016 to 30 September 2019. Patient profiles are shown in Table 1. A diagnosis of HCC was established using histological findings or radiological modalities according to the American Association for the Study of Liver Diseases criteria [52], Child-Pugh class A liver function, Eastern Cooperative Oncology Group performance status [53] of at most 1, and expected survival time higher than 3 months. mRECIST [39] was used to assess the effect of the treatment to the targeted lesion. Up to 5 target organs were selected, and up to 2 of each organ was used as a target lesions in this study. Spider plots and waterfall plots were constructed using the sum of the longest diameters of maximum two target tumors in the liver.

This study was approved by the institutional review board of the Kindai University Hospital, Ethical Code: 21-42, approved in 2015 and the investigators obtained informed consent from each patient. The written informed consent form was provided from the objectives of the study, along with details of the treatment protocol and the dose of each ICI agent and lenvatinib. The potential risks and benefits of the treatments were also discussed with the patients while obtaining consent.

\subsection{Treatment Regimens}

Lenvatinib (Lenvima; Eisai Co., Ltd., Tokyo, Japan) was orally administered as next-line chemotherapy to patients with unresectable HCC who relapsed or were refractory to previous treatment with anti-PD-1/PD-L1 antibody. The doses of lenvatinib were determined according to body weight: patients weighing $<60 \mathrm{~kg}$ received $8 \mathrm{mg}$ once daily, whereas those weighing $\geq 60 \mathrm{~kg}$ received an initial dose of $12 \mathrm{mg}$ once daily. Dose reductions or treatment interruptions were considered on 
a case-by-case basis according to the severity of each AE until symptoms resolved or patient condition returned to baseline.

\subsection{Efficacy Assessment}

Dynamic computed tomography or magnetic resonance imaging was performed every 4-8 weeks, and treatment response was assessed according to mRECIST [39]. ORR was determined by including patients who attained CR and those who attained PR, and the DCR was defined as a sum of ORR and SD. The DCR and ORR were determined with the best response recorded from the beginning of lenvatinib treatment until disease progression or recurrence occurred. For safety assessment, AEs were assessed according to the National Cancer Institute's Common Terminology Criteria for Adverse Events version 4.0 [54].

\subsection{Statistical Analysis}

Patients were followed up until 29 February 2020 or death using medical records and outpatient visits. The primary outcome was PFS, whereas the secondary outcomes were DCR, ORR, and OS. The baseline characteristics and disease factors of patients are expressed as median IQR. PFS and OS were estimated from the time of the initial lenvatinib administration to the occurrence of tumor progression or death by any cause. Each survival time was measured using the Kaplan-Meier method. Analyses were performed using the SPSS software (version 22; SPSS, Chicago, IL, USA).

\section{Conclusions}

Lenvatinib administered to patients with unresectable HCC who were unresponsive to PD-1/PD-L1 checkpoint blockade demonstrated considerable antitumor activity and a tolerable safety profile. However, because of the limited number of the cases analyzed here, additional investigations with larger cohorts are warranted.

Author Contributions: Conceptualization, M.K. and K.U.; Methodology, M.K.; Software, T.A.; Validation, T.A.; Formal Analysis, T.A.; Investigation, T.A. and M.T. (Masahiro Takita); Data Curation, T.A., K.U., M.M., H.C., M.T. (Masahiro Takita), S.H., H.I., Y.M. and N.N.; Writing-Original Draft Preparation, T.A. and Y.M.; Writing-Review and Editing, N.N. and M.K.; Visualization, M.T. (Masakatsu Tsurusaki); Supervision, M.K.; Project Administration, N.N. and M.K. All authors have read and agreed to the published version of the manuscript.

Funding: Japan Society for the Promotion of Science: 18K07922.

Conflicts of Interest: T.A: No relevant conflicts of interest to disclose. M.K. has received grants from Taiho Pharmaceuticals, Chugai Pharmaceuticals, Otsuka, Takeda, Sumitomo Dainippon-Sumitomo, Daiichi Sankyo, AbbVie, Astellas Pharma, and Bristol-Myers Squibb. He has also received grants and personal fees from Merck Sharpe and Dohme (MSD), Eisai, and Bayer, and is an adviser for MSD, Eisai, Bayer, Bristol-Myers Squibb, Eli Lilly, and ONO Pharmaceuticals. K.U. has received honoraria from Eisai, Eli Lilly. M.M., H.C., M.T., S.H., H.I., Y.M., M.T., N.N. and T.A. declare no conflict of interest.

\section{References}

1. El-Serag, H.B.; Rudolph, K.L. Hepatocellular Carcinoma: Epidemiology and Molecular Carcinogenesis. Gastroenterol. 2007, 132, 2557-2576. [CrossRef]

2. Ferlay, J.; Soerjomataram, I.; Dikshit, R.; Eser, S.; Mathers, C.; Rebelo, M.; Parkin, D.M.; Forman, D.; Bray, F. Cancer incidence and mortality worldwide: Sources, methods and major patterns in GLOBOCAN 2012. Int. J. Cancer 2015, 136, E359-E386. [CrossRef]

3. Balogh, J.; Victor, D.; Asham, E.H.; Burroughs, S.G.; Boktour, M.; Saharia, A.; Li, X.; Ghobrial, R.M.; Monsour, H.P. Hepatocellular carcinoma: a review. J. Hepatocell. Carcinoma 2016, 3, 41-53. [CrossRef]

4. Galle, P.R.; Forner, A.; Llovet, J.M.; Mazzaferro, V.; Piscaglia, F.; Raoul, J.L.; Schirmacher, P.; Vilgrain, V. EASL Clinical Practice Guidelines: Management of hepatocellular carcinoma. J. Hepatol. 2018, 69, 182-236. [CrossRef] 
5. Kudo, M.; Izumi, N.; Sakamoto, M.; Matsuyama, Y.; Ichida, T.; Nakashima, O.; Matsui, O.; Ku, Y.; Kokudo, N.; Makuuchi, M.; et al. Survival Analysis over 28 Years of 173,378 Patients with Hepatocellular Carcinoma in Japan. Liver Cancer 2016, 5, 190-197. [CrossRef]

6. Llovet, J.M.; Ricci, S.; Mazzaferro, V.; Hilgard, P.; Gane, E.; Blanc, J.-F.; De Oliveira, A.C.; Santoro, A.; Raoul, J.-L.; Forner, A.; et al. Sorafenib in Advanced Hepatocellular Carcinoma. N. Engl. J. Med. 2008, 359, 378-390. [CrossRef]

7. Cheng, A.-L.; Kang, Y.-K.; Chen, Z.; Tsao, C.-J.; Qin, S.; Kim, J.S.; Luo, R.; Feng, J.; Ye, S.; Yang, T.-S.; et al. Efficacy and safety of sorafenib in patients in the Asia-Pacific region with advanced hepatocellular carcinoma: a phase III randomised, double-blind, placebo-controlled trial. Lancet Oncol. 2009, 10, 25-34. [CrossRef]

8. Kudo, M.; Finn, R.S.; Qin, S.; Han, K.-H.; Ikeda, K.; Piscaglia, F.; Baron, A.; Park, J.-W.; Han, G.; Jassem, J.; et al. Lenvatinib versus sorafenib in first-line treatment of patients with unresectable hepatocellular carcinoma: a randomised phase 3 non-inferiority trial. Lancet 2018, 391, 1163-1173. [CrossRef]

9. Bruix, J.; Qin, S.; Merle, P.; Granito, A.; Huang, Y.-H.; Bodoky, G.; Pracht, M.; Yokosuka, O.; Rosmorduc, O.; Breder, V.; et al. Regorafenib for patients with hepatocellular carcinoma who progressed on sorafenib treatment (RESORCE): a randomised, double-blind, placebo-controlled, phase 3 trial. Lancet 2017, 389, 56-66. [CrossRef]

10. Zhu, A.X.; Kang, Y.-K.; Yen, C.-J.; Finn, R.S.; Galle, P.R.; Llovet, J.M.; Assenat, E.; Brandi, G.; Pracht, M.; Lim, H.Y.; et al. Ramucirumab after sorafenib in patients with advanced hepatocellular carcinoma and increased $\alpha$-fetoprotein concentrations (REACH-2): A randomised, double-blind, placebo-controlled, phase 3 trial. Lancet Oncol. 2019, 20, 282-296. [CrossRef]

11. Abou-Alfa, G.K.; Meyer, T.; Cheng, A.-L.; El-Khoueiry, A.B.; Rimassa, L.; Ryoo, B.-Y.; Cicin, I.; Merle, P.; Chen, Y.; Park, J.-W.; et al. Cabozantinib in Patients with Advanced and Progressing Hepatocellular Carcinoma. N. Engl. J. Med. 2018, 379, 54-63. [CrossRef]

12. Bouattour, M.; Mehta, N.; He, A.R.; Cohen, E.I.; Nault, J.-C. Systemic Treatment for Advanced Hepatocellular Carcinoma. Liver Cancer 2019, 8, 341-358. [CrossRef]

13. Rimassa, L.; Pressiani, T.; Merle, P. Systemic Treatment Options in Hepatocellular Carcinoma. Liver Cancer 2019, 8, 427-446. [CrossRef]

14. Matsui, J.; Funahashi, Y.; Uenaka, T.; Watanabe, T.; Tsuruoka, A.; Asada, M. Multi-Kinase Inhibitor E7080 Suppresses Lymph Node and Lung Metastases of Human Mammary Breast Tumor MDA-MB-231 via Inhibition of Vascular Endothelial Growth Factor-Receptor (VEGF-R) 2 and VEGF-R3 Kinase. Clin. Cancer Res. 2008, 14, 5459-5465. [CrossRef]

15. Matsui, J.; Yamamoto, Y.; Funahashi, Y.; Tsuruoka, A.; Watanabe, T.; Wakabayashi, T.; Uenaka, T.; Asada, M. E7080, a novel inhibitor that targets multiple kinases, has potent antitumor activities against stem cell factor producing human small cell lung cancer H146, based on angiogenesis inhibition. Int. J. Cancer 2007, 122, 664-671. [CrossRef]

16. Yamada, K.; Yamamoto, N.; Yamada, Y.; Nokihara, H.; Fujiwara, Y.; Hirata, T.; Koizumi, F.; Nishio, K.; Koyama, N.; Tamura, T. Phase I Dose-Escalation Study and Biomarker Analysis of E7080 in Patients with Advanced Solid Tumors. Clin. Cancer Res. 2011, 17, 2528-2537. [CrossRef]

17. Boss, D.S.; Glen, H.; Beijnen, J.H.; Keesen, M.; Morrison, R.; Tait, B.; Copalu, W.; Mazur, A.; Wanders, J.; O'Brien, J.P.; et al. A phase I study of E7080, a multitargeted tyrosine kinase inhibitor, in patients with advanced solid tumours. Br. J. Cancer 2012, 106, 1598-1604. [CrossRef]

18. Boku, N.; Ryu, M.-H.; Kato, K.; Chung, H.; Minashi, K.; Lee, K.-W.; Cho, H.; Kang, W.; Komatsu, Y.; Tsuda, M.; et al. Safety and efficacy of nivolumab in combination with S-1/capecitabine plus oxaliplatin in patients with previously untreated, unresectable, advanced, or recurrent gastric/gastroesophageal junction cancer: Interim results of a randomized, phase II trial (ATTRACTION-4). Ann. Oncol. 2019, 30, 250-258. [CrossRef]

19. Gandhi, L.; Rodríguez-Abreu, D.; Gadgeel, S.; Esteban, E.; Felip, E.; De Angelis, F.; Domine, M.; Clingan, P.; Hochmair, M.J.; Powell, S.F.; et al. Pembrolizumab plus Chemotherapy in Metastatic Non-Small-Cell Lung Cancer. N. Engl. J. Med. 2018, 378, 2078-2092. [CrossRef]

20. Socinski, M.A.; Jotte, R.M.; Cappuzzo, F.; Orlandi, F.; Stroyakovskiy, D.; Nogami, N.; Rodríguez-Abreu, D.; Moro-Sibilot, D.; Thomas, C.A.; Barlesi, F.; et al. Atezolizumab for First-Line Treatment of Metastatic Nonsquamous NSCLC. N. Engl. J. Med. 2018, 378, 2288-2301. [CrossRef]

21. Nishida, N.; Kudo, M. Immune checkpoint blockade for the treatment of human hepatocellular carcinoma. Hepatol. Res. 2018, 48, 622-634. [CrossRef] 
22. Kudo, M. Immuno-Oncology Therapy for Hepatocellular Carcinoma: Current Status and Ongoing Trials. Liver Cancer 2019, 8, 221-238. [CrossRef]

23. El-Khoueiry, A.B.; Sangro, B.; Yau, T.; Crocenzi, T.S.; Kudo, M.; Hsu, C.; Kim, T.-Y.; Choo, S.-P.; Trojan, J.; Welling, T.H.; et al. Nivolumab in patients with advanced hepatocellular carcinoma (CheckMate 040): An open-label, non-comparative, phase 1/2 dose escalation and expansion trial. Lancet 2017, 389, 2492-2502. [CrossRef]

24. Zhu, A.X.; Finn, R.S.; Edeline, J.; Cattan, S.; Ogasawara, S.; Palmer, D.; Verslype, C.; Zagonel, V.; Fartoux, L.; Vogel, A.; et al. Pembrolizumab in patients with advanced hepatocellular carcinoma previously treated with sorafenib (KEYNOTE-224): A non-randomised, open-label phase 2 trial. Lancet Oncol. 2018, 19, 940-952. [CrossRef]

25. Yau, T.; Park, J.W.; Finn, R.S.; Cheng, A.; Mathurin, P.; Edeline, J.; Kudo, M.; Han, K.; Harding, J.J.; Merle, P.; et al. CheckMate 459: A Randomized, Multi-Center Phase 3 Study of Nivolumab vs Sorafenib as First-Line Treatment in Patients with Advanced Hepatocellular Carcinoma. Ann. Oncol. 2019, 30, v851-v934. [CrossRef]

26. Finn, R.S.; Ryoo, B.-Y.; Merle, P.; Kudo, M.; Bouattour, M.; Lim, H.Y.; Breder, V.; Edeline, J.; Chao, Y.; Ogasawara, S.; et al. Pembrolizumab As Second-Line Therapy in Patients With Advanced Hepatocellular Carcinoma in KEYNOTE-240: A Randomized, Double-Blind, Phase III Trial. J. Clin. Oncol. 2020, 38, $193-202$. [CrossRef]

27. Finn, R.S.; Qin, S.; Ikeda, M.; Galle, P.R.; Ducreux, M.; Kim, T.-Y.; Kudo, M.; Breder, V.; Merle, P.; Kaseb, A.O.; et al. Atezolizumab plus Bevacizumab in Unresectable Hepatocellular Carcinoma. N. Engl. J. Med. 2020, 382, 1894-1905. [CrossRef]

28. Morais, C. Sunitinib resistance in renal cell carcinoma. J. Kidney Cancer VHL 2014, 1, 1-11. [CrossRef]

29. Teng, M.W.L.; Ngiow, S.F.; Ribas, A.; Smyth, M.J. Classifying Cancers Based on T-cell Infiltration and PD-L1. Cancer Res. 2015, 75, 2139-2145. [CrossRef]

30. Sia, D.; Jiao, Y.; Martinez-Quetglas, I.; Kuchuk, O.; Villacorta-Martin, C.; De Moura, M.C.; Putra, J.; Camprecios, G.; Bassaganyas, L.; Akers, N.; et al. Identification of an Immune-specific Class of Hepatocellular Carcinoma, Based on Molecular Features. Gastroenterol. 2017, 153, 812-826. [CrossRef]

31. Kudo, M. Scientific Rationale for Combined Immunotherapy with PD-1/PD-L1 Antibodies and VEGF Inhibitors in Advanced Hepatocellular Carcinoma. Cancers 2020, 12, 1089. [CrossRef]

32. Finn, R.S.; Ikeda, M.; Zhu, A.X.; Sung, M.W.; Baron, A.D.; Kudo, M.; Okusaka, T.; Kobayashi, M.; Kumada, H.; Kaneko, S.; et al. Phase Ib Study of Lenvatinib Plus Pembrolizumab in Patients With Unresectable Hepatocellular Carcinoma. J. Clin. Oncol. 2020, 38, 2960-2970. [CrossRef]

33. Osa, A.; Uenami, T.; Koyama, S.; Fujimoto, K.; Okuzaki, D.; Takimoto, T.; Hirata, H.; Yano, Y.; Yokota, S.; Kinehara, Y.; et al. Clinical implications of monitoring nivolumab immunokinetics in non-small cell lung cancer patients. JCI Insight 2018, 3. [CrossRef]

34. Kudo, M. Extremely High Objective Response Rate of Lenvatinib: Its Clinical Relevance and Changing the Treatment Paradigm in Hepatocellular Carcinoma. Liver Cancer 2018, 7, 215-224. [CrossRef]

35. Mittal, D.; Gubin, M.M.; Schreiber, R.D.; Smyth, M.J. New insights into cancer immunoediting and its three component phases—elimination, equilibrium and escape. Curr. Opin. Immunol. 2014, 27, 16-25. [CrossRef]

36. Garcia-Diaz, A.; Shin, D.S.; Moreno, B.H.; Saco, J.; Escuin-Ordinas, H.; Rodriguez, G.A.; Zaretsky, J.M.; Sun, L.; Hugo, W.; Wang, X.; et al. Interferon Receptor Signaling Pathways Regulating PD-L1 and PD-L2 Expression. Cell Rep. 2017, 19, 1189-1201. [CrossRef]

37. Corrigendum to "EASL Clinical Practice Guidelines: Management of hepatocellular carcinoma" [J Hepatol 69 (2018) 182-236]. J. Hepatol. 2019, 70, 817. [CrossRef]

38. Sterling, R.K.; Lissen, E.; Clumeck, N.; Sola, R.; Correa, M.C.; Montaner, J.; Sulkowski, M.S.; Torriani, F.J.; Dieterich, D.T.; Thomas, D.L.; et al. Development of a simple noninvasive index to predict significant fibrosis in patients with HIV/HCV coinfection. Hepatology 2006, 43, 1317-1325. [CrossRef]

39. Lencioni, R.; Llovet, J.M. Modified RECIST (mRECIST) Assessment for Hepatocellular Carcinoma. Semin. Liver Dis. 2010, 30, 052-060. [CrossRef]

40. Kudo, M. A New Era in Systemic Therapy for Hepatocellular Carcinoma: Atezolizumab plus Bevacizumab Combination Therapy. Liver Cancer 2020, 9, 1-19. [CrossRef]

41. Xie, Q.-K.; Zhao, Y.-J.; Pan, T.; Lyu, N.; Mu, L.-W.; Li, S.-L.; Shi, M.-D.; Zhang, Z.-F.; Zhou, P.-H.; Zhao, M. Programmed death ligand 1 as an indicator of pre-existing adaptive immune responses in human hepatocellular carcinoma. OncoImmunology 2016, 5, e1181252. [CrossRef] 
42. De Galarreta, M.R.; Bresnahan, E.; Molina-Sánchez, P.; Lindblad, K.E.; Maier, B.; Sia, D.; Puigvehi, M.; Miguela, V.; Casanova-Acebes, M.; Dhainaut, M.; et al. $\beta$-Catenin Activation Promotes Immune Escape and Resistance to Anti-PD-1 Therapy in Hepatocellular Carcinoma. Cancer Discov. 2019, 9, 1124-1141. [CrossRef]

43. Pinyol, R.; Sia, D.; Llovet, J.M. Immune Exclusion-Wnt/CTNNB1 Class Predicts Resistance to Immunotherapies in HCC. Clin. Cancer Res. 2019, 25, 2021-2023. [CrossRef]

44. Harding, J.J.; Nandakumar, S.; Armenia, J.; Khalil, D.N.; Albano, M.; Ly, M.; Shia, J.; Hechtman, J.F.; Kundra, R.; El Dika, I.; et al. Prospective Genotyping of Hepatocellular Carcinoma: Clinical Implications of Next-Generation Sequencing for Matching Patients to Targeted and Immune Therapies. Clin. Cancer Res. 2018, 25, 2116-2126. [CrossRef]

45. Paterson, A.C.; Sciot, R.; Kew, M.C.; Callea, F.; Dusheiko, G.M.; Desmet, V.J. HLA expression in human hepatocellular carcinoma. Br. J. Cancer 1988, 57, 369-373. [CrossRef]

46. Berko, D.; Carmi, Y.; Cafri, G.; Ben-Zaken, S.; Sheikhet, H.M.; Tzehoval, E.; Eisenbach, L.; Margalit, A.; Gross, G. Membrane-Anchored $\beta 2$-Microglobulin Stabilizes a Highly Receptive State of MHC Class I Molecules. J. Immunol. 2005, 174, 2116-2123. [CrossRef]

47. Tohyama, O.; Matsui, J.; Kodama, K.; Hata-Sugi, N.; Kimura, T.; Okamoto, K.; Minoshima, Y.; Iwata, M.; Funahashi, Y. Antitumor Activity of Lenvatinib (E7080): An Angiogenesis Inhibitor That Targets Multiple Receptor Tyrosine Kinases in Preclinical Human Thyroid Cancer Models. J. Thyroid. Res. 2014, 2014, 1-13. [CrossRef]

48. Yamauchi, M.; Ono, A.; Ishikawa, A.; Kodama, K.; Uchikawa, S.; Hatooka, H.; Zhang, P.; Teraoka, Y.; Morio, K.; Fujino, H.; et al. Tumor Fibroblast Growth Factor Receptor 4 Level Predicts the Efficacy of Lenvatinib in Patients With Advanced Hepatocellular Carcinoma. Clin. Transl. Gastroenterol. 2020, 11, e00179. [CrossRef]

49. Evoron, T.; Emarcheteau, E.; Epernot, S.; Ecolussi, O.; Tartour, E.; Etaieb, J.; Eterme, M. Control of the Immune Response by Pro-Angiogenic Factors. Front. Oncol. 2014, 4, 70. [CrossRef]

50. Shigeta, K.; Datta, M.; Hato, T.; Kitahara, S.; Chen, I.X.; Matsui, A.; Kikuchi, H.; Mamessier, E.; Aoki, S.; Ramjiawan, R.R.; et al. Dual Programmed Death Receptor-1 and Vascular Endothelial Growth Factor Receptor-2 Blockade Promotes Vascular Normalization and Enhances Antitumor Immune Responses in Hepatocellular Carcinoma. Hepatology 2019, 71, 1247-1261. [CrossRef]

51. Kato, Y.; Tabata, K.; Kimura, T.; Yachie-Kinoshita, A.; Ozawa, Y.; Yamada, K.; Ito, J.; Tachino, S.; Hori, Y.; Matsuki, M.; et al. Lenvatinib plus anti-PD-1 antibody combination treatment activates CD8+ T cells through reduction of tumor-associated macrophage and activation of the interferon pathway. PLOS ONE 2019, 14, e0212513. [CrossRef] [PubMed]

52. Marrero, J.A.; Kulik, L.M.; Sirlin, C.B.; Zhu, A.X.; Finn, R.S.; Abecassis, M.; Roberts, L.R.; Heimbach, J.K. Diagnosis, Staging, and Management of Hepatocellular Carcinoma: 2018 Practice Guidance by the American Association for the Study of Liver Diseases. Hepatology 2018, 68, 723-750. [CrossRef] [PubMed]

53. Oken, M.M.; Creech, R.H.; Tormey, D.C.; Horton, J.; Davis, T.E.; McFadden, E.T.; Carbone, P.P. Toxicity and response criteria of the Eastern Cooperative Oncology Group. Am. J. Clin. Oncol. 1982, 5, 649-656. [CrossRef] [PubMed]

54. Cancer Therapy Evaluation Program. Available online: https:/ctep.cancer.gov/protocolDevelopment/ electronic_applications/ctc.htm (accessed on 21 March 2017).

(C) 2020 by the authors. Licensee MDPI, Basel, Switzerland. This article is an open access article distributed under the terms and conditions of the Creative Commons Attribution (CC BY) license (http://creativecommons.org/licenses/by/4.0/). 